\title{
An ultrasound-guided percutaneous nerve stimulation regimen based on finite element modeling for median nerve rupture
}

xiaolei chu ( $\nabla$ chuxiaolei8@163.com )

Tianjin University

\section{Xizi Song}

Tianjin University

\section{Yuru Li}

Tianjin University of Sport

\section{Ziren Wu}

Tianjin University of Sport

Qi Li

Tianjin Hospital

\section{Qingwen Li}

Tianjin University of Sport

\section{Xiaosong Gu}

Tianjin University

\section{Dong Ming}

Tianjin University

\section{Research}

Keywords: Peripheral nerve injury, percutaneous nerve stimulation, median nerve rupture, finite element modeling

Posted Date: November 15th, 2021

DOI: https://doi.org/10.21203/rs.3.rs-1063940/v1

License: (c) (i) This work is licensed under a Creative Commons Attribution 4.0 International License. Read Full License 


\section{Abstract}

Object: In order to promote the functional recovery of median nerve rupture patients, this paper proposes an ultrasound-guided percutaneous nerve stimulation regimen based on finite element modeling.

Method: First, according to anatomy feature, a multi-layer human forearm model is constructed. What's more, taking current density and activate function as optimization indicators, the percutaneous nerve stimulation regimen is designed with finite element modeling, including electrical needle angel, distance and position. Finally, to test the performance of designed regimen, a total of 22 patients with median nerve rupture participate in the clinical randomized controlled trial. And the clinical treatment effect is evaluated with BMRC, grip strength, functional score of median nerve and DASH scores.

Results: The designed percutaneous nerve stimulation regimen is that, parallel to each other, electrical needles are located at both ends of the injury nerve with a distance of $3 \mathrm{~cm}$. Besides, clinical trial results show that, after treatment the difference in sensory function was statistically significant. In terms of motor function, the BMRC of motor and grip strength are improved in both groups after treatment and there are significant differences between groups. Besides the grip strength improvement of experimental group is 4 times higher than that of control group. On global function, the DASH scores are reduced by $29 \%$ with experimental group but only $8 \%$ with control group; functional score of median nerve improved by $24 \%$ in the experimental group and by $22 \%$ in the control group, but the difference between groups is statistically significant after treatment.

Conclusion: Clinical trial results demonstrate that the designed percutaneous nerve stimulation regimen can significantly improve the sensory, motor and global function of median nerve rupture, which is a potential clinical treatment regimen.

Trial registration: ChiCTR, ChiCTR2000030790. Registered14 march 2020, http://www.chictr.org.cn/ChiCTR2000030790

\section{Introduction}

The median nerve, also known as the 'eye of the hand,' is a mixed nerve that plays plays a crucial role in the functionality of the hand. If the median nerve is damaged, the ability to abduct and oppose the thumb may be lost due to paralysis of the thenar muscles, resulting in severe dysfunction that severely affects the activities of daily living (Robinson, 2000; Wang et al., 2019). It is more common to suffer neurotmesis at the forearm or wrist as the course of the nerve lies more superficial in these areas. The characteristic of neurotmesis is complete disconnection between the two segments of the injured nerve. Hence, there is complete loss of function and recovery without surgical intervention. However, neurotmesis is often associated with poor outcomes even after traditional surgical techniques.

In general, pharmacological treatment should take precedence over surgery. Many studies have been indicated that various medicines can enhance and promote nerve regeneration after neurotmesis. 
However, the use of pharmacological treatment locally appears appears to be more effective than systemic application, but its effectiveness is limited by rapid degradation and absorption(Suslu et al., 2013). But continuous systemic administrations cause severe secondary metabolic effects, and there is no method to overcome these problems (Mukudai et al., 2015). In recent years, more and more scholars began to pay attention to the role of cell-based therapies in peripheral nerve injury (PNI), and several scholars considered this method as a potential alternative treatment. Nevertheless, according to the current research, this treatment method still has a certain risk and challenge. It is traumatic both for the cells and intraneural architecture to inject suspension of (mesenchymal stem cells) at the lesion site which can lead to a defective cellular distribution(Pang et al., 2013).

Electrical stimulation (ES) is regarded as effective physical therapy for accelerating nerve regeneration and functional recovery, and several researchers widely explored and studied its application. Recent research demonstrated that ES in the proximal nerve stump with low intensity can promote nerve regeneration (Lal et al., 2008; Kim et al., 2011) by increasing the expression of brain derived neurotrophic factor (BDNF)(Al-Majed et al., 2000a). At the same time, the proliferation of Schwann cells and the formation of myelin sheath were promoted during regeneration (Wan et al., 2010; Zhang et al., 2013). In addition, promoting the expression of genes associated with growth factors (Geremia et al., 2007) and neutrophine signalling (English et al., 2007) may also be one of the therapeutic mechanisms of ES. According to the depth of the electrodes, we divided the stimulation into transcutaneous electrical nerve stimulation (TENS), subcutaneous electrical stimulation (SQS) and peripheral nerve stimulation (PNS). TENS ' electrical stimulation electrodes are placed on the skin and electrical impulses are applied to stimulate the endings of the nervous system.

The characteristic of the SQS is placing electrical stimulation electrodes in the subcutaneous spaces. However, the electrodes of PNS usually surround the peripheral nerves. Low-frequency TENS immediately after injury have been verified can improve peripheral nerve regeneration (Cavalcante Miranda de Assis et al., 2014). Several studies compared the TENS and PNS methods. They found that the intensity around the sciatic nerve was significantly higher in the PNS group than in the TENS group. This may be due to the greater concentration and parallel distribution of the electric field relative to the target nerve (Ju et al., 2020). PNS now typically relies on the implantation of a cuff electrode around the nerve. An expensive operation is needed to implant the electrode around the nerve. Although PNS is appreciated for its high precision and targeting, its complications and cost discourage participation.

SQS has deeper electrical stimulation ability than TENS. Plus, it does not require an operation to implant an electrode. A study compared implanted electrodes with 4 kinds of contacts in the resected sciatic nerve (point contact; $1 / 4$ circle contact; whole-circle contact; and no electrodes as control). They found that point contact has the same effect as the whole-circle contact (Yu et al., 2019). In this way, we can speculate that electroacupuncture (EA), as a kind of SQS, is a low-cost and valid therapy. In China, the mechanism of SQS has been extensive studied, which is a therapy that combines the use of acupuncture points and needles that conduct electrical current to produce therapeutic effects in human subjects and experimental animals. However, electrical needles often select points according to acupuncture or jingluo. 
There are various acupunctures that are reported to treat peripheral nerve injury. It is hard to acquire a consistent response to electrical needle stimulation since differences in electrode placement, skin movement, and physiological variables affecting tissue conductance. This requires a feasible clinical regimen that must be able to adapt to these changes in order to produce repeatable results. So some computer models were developed in order to explore the effects of these variations more fully and accurately, and to avoid exhaustive human testing.

Therefore, the finite element model is established in this study on the basis of existing models in the literature, and verified by experimental data(Hassan et al., 2018a). This model is helpful to predict the influence of different electrode distance, angle and position on the activation degree of peripheral nerve axons. Finally, we get an optimized regimen for median nerve neurotmesis according to the compute model from Comsole software. Then the randomized and parallel-group clinical trial is considered the optimal study design to assess the efficacy. We hypothesis the regimen from the Comsole software can improve the transection injury of the medial nerve regeneration and functional recovery. Further, we are currently enrolling patients with the PNI to confirm the efficacy of regimen.

\section{Materials And Methods}

The study is divided into two-steps. The first stage is building a three-dimensional finite element model (FEM) of the forearm according anatomy. In this model, electrical properties are assigned to each tissue and electrode. This forearm model is used to predict the current density and activation function of the entire nerve tract within the median nerve. Then simulation is performed to explore percutaneous nerve stimulation regimen including angel, distant and location of the electrodes. The second step, this regimen is validated with clinical randomized controlled trial data.

\section{Finite element model}

A 3-dimensional forearm model is created by finite element software (Comsole Multiphysics, Comsole Inc) which the length is $20 \mathrm{~cm}$ along the longitudinal axis (Supplementary 1). Based on gross dissections conducted, we modeled the median as a single monofascicular nerve with a diameter of $2 \mathrm{~mm}$. The geometry of the human forearm is modeled by concentric cylinders for the skin $(3 \mathrm{~mm})$, fat $(10 \mathrm{~mm})$, muscle $(25 \mathrm{~mm})$, bone $(2 \mathrm{~cm})$ and blood vessel $(3 \mathrm{~mm})$ as it is presented in Figure 1(Hassan et al., 2018b). The corresponding tissue layer is described by the conductivity $\sigma$ and also the relative permittivity $\varepsilon$. We refer to Gabriel et al. 's study for $\sigma$ and $\varepsilon$ for each component of the model as shown in Supplementary 2 (Gabriel et al., 1996).

Percutaneous nerve stimulation is modeled using two un-insulated stainless steel needle electrodes (cylinder, diameter $=0.3 \mathrm{~mm}$, length $=40 \mathrm{~mm}$ ). Simulations are run under conditions of constant prescribed current $(0.3 \mathrm{~V})$. The electrical stimulation frequency is $20 \mathrm{~Hz}$, which is believed to effectively promote nerve regeneration (Gordon et al., 2009; Park et al., 2019). Percutaneous nerve stimulation is simulated by changing the position of the electrodes, leaving all other parameters of the model 
unchanged. Next solving the FEM yielded to calculate the current density and active function of the corresponding nerve fibers. The current density represents the current per unit section area. The second derivative of biological tissue's electrical potential along the nerve fiber axis under the action of electrical stimulation is called active function which can characterize the effect of electric field on peripheral nerve electrical activity (McNeal, 1976; Rattay and Aberham, 1993). When neurons depolarize and excite, the active function is positive; in contrast, when neurons hyperpolarized and inhibited excitability, the excitation function was negative. In order to calculate the best location for two electrodes, we set three lines in the model: center line of nerve, forearm line of nerve and line between two needles (Figure 1). The central line of the nerve is regarded the key index. We determine the percutaneous nerve stimulation regimen mainly by the ratio of the value between the forearm line of nerve and line between two needles.

\section{Clinical trial}

\subsection{Participants}

Patients diagnosed with median nerve neurotmesis (aged from 10 to 70 years) are recruited from Tianjin hospital rehabilitation department. The inclusion and exclusion criteria are set out below. Inclusion criteria: 1) patients who were diagnosed with median nerve rupture based on clinical symptoms and physical examinations with confirmation by nerve conduction studies; 2)patients accepted neurorrhaphy and vital signs are stable; 3 ) disease course $<2$ months; 4) NULL of the patients had previously undergone electrical stimulation treatment and no skin allergy to electrical stimulation. Exclusion criteria: 1) patients with diseases of important organs, such as heart disease, hypertension, tuberculosis, asthma, hepatitis, and nephritis; 2) Subjects with serious metabolic diseases (such as hyperthyroidism, hypothyroidism and edema, etc.) or chronic use of drugs affecting metabolism were excluded; 3) acute patients who have suffered from high fever, diarrhea and other acute diseases in the near future (within 1 month), and have not recovered their physical strength; 4) unstable fractures of the upper extremity.

Altogether, 21 participants were included in the present study and randomly assigned to an experimental $(n=12)$ and a control group $(n=10)$ in a randomized two-group design. Moreover, one of the patients in control has two-arm median nerve recruitment broken.

\subsection{Method}

Patients in the control group received conventional rehabilitation treatment and TENS, while those in the experimental group received both conventional rehabilitation treatment and percutaneous nerve stimulation. Conventional rehabilitation treatment including 1) Movement training: passive and active extend/flexible of fingers, elbows and shoulders, aiming at preventing joint ankylosis and muscle antrophy; 2) Manipulative therapy: the aim is reliving muscle cramp, eliminating local swell, releasing adhesion and reliving a series of clinical symptoms such as pain, chills, numbness; 3 ) Occupational therapy: such as picking up glass balls and so on; 4) Sensory training: including cold and hot sensory training, two-point discrimination training and positioning training. Patients receive conventional rehabilitation once a day, 1 hour per session, 4 times a week, for 5 consecutive weeks. Percutaneous 
nerve stimulation: firstly, locate the site of median nerve recruitment and measure the distance from skin to nerve by SonoScape-X5 ultrasound (Shenzhen SonoScape Bio-Medical Technology Co. Ltd., Shenzhen, China) (Supplementary 3). Then two disposables, sterilized stainless-steel acupuncture needles (0.3*40mm, Beijing Keyuanda Medical Device Manufactory, Beijing, China) are used for percutaneous nerve stimulation. The stimulation apply with Electronic Acupuncture Instrument (SDZ-III, Hwato, Suzhou, China) with discontinuous wave (frequency $20 \mathrm{~Hz}$; width: $0.2 \mathrm{~ms}$ ) for 1 hour per session, once a day, 4 times a week, for 5 consecutive (Supplementary 4). TENS: The sites of breakage locate at the middle of the two electrodes and electrodes are attached at the same locations and under identical conditions in all patients (Supplementary 5).

\subsection{Outcome measurements}

\subsubsection{Motor Functional Assessment}

Among the indices of motor functional assessment, the British Medical Research Council (BMRC) has been adopted by most practitioners. It was modified by MacKinnon and Dellon in 1988 and used for assessing sensory and motor function after PNI (Lundborg et al., 2004). BMRC is the most effective method for evaluating the function of a single nerve innervating both proximal and distal muscles (Shen and Zhu, 1996). According assessment motor function is divided six grades from M0 to M5. A higher grade indicates a better level of functioning.

However, using BMRC to evaluate the motor function is highly subjective and the classification is rough. Indeed, motor function tightly correlates with muscle strength. When the target muscle denervation, hand strength will be reduced. The denervation of target skeletal muscles occurs following PNI, showing muscle weakness. Grip strength need activate extrinsic and intrinsic hand muscles, of which the forearm peripheral nerve-innervated muscles are a major contributor (Power et al., 2020). Schreuders et al found that the strength will decrease $32 \%$ than normal people when median nerve is blocked (Schreuders et al., 2000; Wachter et al., 2018a). Based on this, some scholars further corroborated grip strength could be implemented as a diagnostic tool for median nerve injury rehabilitation (Wachter et al., 2018b). Therefore, we focus on evaluating motor innervation by measuring grip strength before and after treatment. We assess grip strength using a hydraulic hand dynamometer (Baseline ${ }^{\circledR} \mathrm{HiRes}^{\mathrm{TM}} \mathrm{ER}^{\mathrm{TM}}$, USA).

\subsubsection{Sensory Functional Assessment}

In sensory innervation, the median nerve distribution includes the radial direction of the palm, the thumb and index finger, and half of the middle and ring fingers (Soldado et al., 2016). Consequently, sensory dysfunction also is a common complication in patients who have suffered PNI. In our study, we apply the BMRC sensory evaluation index and divided it into eight grades (SO-S4) (Shen and Zhu, 1996). The grade of sensory function is assessed according the deep sensation, superficial sensory and allodynia. A higher grade indicates a better level of functioning.

\subsubsection{Global Function}




\section{Functional Score of Median Nerve}

Upper limb functions assessment was developed by Chinese Society of Hand Surgery in 2000. And the functional score of median nerve is a subfactor of the scale. Median function assessment is performed using this scale (Pan et al., 2000). On the one hand this method does not require any special equipment and is easy operation, on the other hand it also requires as accurately and objectively as possible. The evaluation criteria contain four indicators, including the muscle strength of wrist flexor muscle, flexor digitorum, opposition of the thumb and sensory. Each indicator is divided four levels. Therapist evaluates patients' function by their performance with a minimum score of 0 and a maximum score of 16 points. The higher the score, the better the median nerve function.

\section{Disability of Arm Shoulder and Hand (DASH) Scores}

Hudak et al. screened 150 activities of daily life for many times. Their selected 30 indicators which could best reflect the activity function of patients to form the DASH evaluation table (Hudak et al., 1996). This is a self-reported questionnaire used to assess upper limb function and disability. The scale is divided into two parts: one part includes activities of daily living; the other part reflects the effect of upper limb symptoms on sleep and self-Satisfaction. Each item is rated on a 5-point (1-5). The formulas are as listed below: DASH score $=($ Raw score-30)/1.2. The higher the total score is, the worse the upper limb function. Some study evaluated patient-reported outcome and the factors associated with disability following an upper limb PNI and found it has high relationship between PNI and DASH scores and higher pain, older age, and brachial plexus injury can predict the DASH score (Novak et al., 2009). Hence, we use DASH as an assessment tool to assess patients' upper limb function.

The patients are evaluated before and after therapy and the score of each evaluation is recorded timely. In order to avoid subjective errors, patients are examined by the same physiatrist before and after the treatment.

\section{Statistical Analysis}

All statistical analyses are conducted using SPSS 22.0 (IBM Corp., Armonk, NY, USA). Nonparametric data are analyzed by the Mann-Whitney rank sum test. Measurement data are expressed by mean $\pm S D$, intergroup differences are analyzed by independent sample $t$-tests, and intra-group differences by paired $t$ tests. Categorical data were compared with the Wilcoxon signed-rank test. $\mathrm{P}<0.05$ is considered statistically significant.

\section{Ethics}

The clinical trial involved in this study was approved by the Human Research Ethics Committee of Tianjin Hospital. All patients voluntarily signed written informed consent. This randomized controlled trial has been registered in the Chinese Clinical Trial Registry (ChiCTR2000030790). 


\section{Results}

\section{Result of finite element model}

\subsection{The location of the needles}

The research of Tessa Gordon (Gordon et al., 2010) find the proximal nerve stimulation can promote nerve regeneration. Brushart et al also find that application of $20 \mathrm{~Hz}$ ES to the proximal nerve for 1 hour can temporally compress the staggered regeneration(Brushart et al., 2002). They also explore the possible mechanism for this effect. They find ES can synchronize the distal sump reinnervation. The number of regenerated axons cannot cross nerve transection and suture is increased by the ES. Nonetheless, the jahyun Koo et al think that placing damaged nerve between the two needles also could improve nerve regeneration and lead to better functional outcomes (Koo et al., 2018; Zhang et al., 2018). $A$ research uses EA to stimulate the injury site from the both end of the peripheral nerve. When Schwann cells exposure promoted directed migration, it travels towards the cathode at a mean rate of 7.5 $\mu \mathrm{m} / \mathrm{h}$ (Bunn et al., 2019). EA may promote enhanced sciatic nerve function recovery and SC proliferation by down-regulating miR-1b(Liu et al., 2020). At present, there is no definitive agreement on which of these regimens is more effective. Consequently, a better site between needle and damaged nerve is worth exploring. Moreover, in this regard, majority of studies have been carried out from physiological, but not electrical perspective. On that account, we use the finite element method to compare which is the optimal location from electrical perspective (Figure 2-A and Figure 2-B). The result show if place damaged nerve between the two needles the current density increase $131.54 \%$ compared to the proximal site. Besides, the injury nerve of active function between the needle is 9.47 and the proximal injury nerve of the needle is -7.06 (Figure 2-C), which implies that the former promote neural excitation but the latter is suggestive of neural inhibition. It also confirms the needle between injury sites is the better location.

\subsection{The distance between the needles}

As two needles should be placed at both ends of the nerve injury, we still need to find the right distance between the needles. Then we compare the different distance $(1 \mathrm{~cm}, 2 \mathrm{~cm}, 3 \mathrm{~cm}, 4 \mathrm{~cm}, 5 \mathrm{~cm}, 6 \mathrm{~cm}, 7 \mathrm{~cm}, 8 \mathrm{~cm}$, $9 \mathrm{~cm}, 10 \mathrm{~cm}, 11 \mathrm{~cm}$, and $12 \mathrm{~cm})$. The result show the current density gradually declined with increased distance (Figure 2-D). It can be seen from figure, the value increase significantly when the distance between the needles is $1-3 \mathrm{~cm}$. To further explore the possible reasons, we observe the current density distributions between the two electrodes at different distance. If the distance is too small the electric current will pass from the anode to cathode at once. The nerve will not get enough electric current. However, if anode is far from cathode the electric current will decline gradually and the injury nerve still cannot get enough electric current. As shown in supplementary 6, when the distance between two electrodes is $3 \mathrm{~cm}$ show a moderate current density. Later, we also compare the different needle distance and find the active function of $3-5 \mathrm{~cm}$ distance can achieve the top site (Figure 2-E). Combined with the above current density results, we concluded that $3 \mathrm{~cm}$ is the better distance. To further validate the results above, current distributions with different distance is simulated by a finite element method 
(Supplementary 7). We find that the current is parallel to the nerve when the distance is $3 \mathrm{~cm}$. Several studies have reported that parallel field is more nerve sensitive than orthogonal field (Ju et al., 2020) which may be the reason $3 \mathrm{~cm}$ can get best active function. Therefore we combine the current density and active function and think that $3 \mathrm{~cm}$ is the better distance.

\subsection{The angle between two needles}

Besides, the angle also needs to be noted here. We compare $0-90^{\circ}$ and find when the angle of needles $0^{\circ}, 15^{\circ}, 30^{\circ}, 45^{\circ}$ and $90^{\circ}$ have relatively large current density (Figure 3-A). Then we consider the active function and find $0^{\circ}, 60^{\circ}, 75^{\circ}$ and $90^{\circ}$ is better choice (Figure 3-B). After comprehensive consideration, we think $0^{\circ}$ and $90^{\circ}$ are optimal choice. However, $90^{\circ}$ is harder to accept due to needle is inserted into the skin in a parallel way. Then 0 is the best choice. As shown in Supplementary 8, we further made a distribution map for the current density under the three angles for analysis. The image intuitively shows that the current density distribution was the most uniform and the current direction is parallel to the nerve when the angle is $0^{\circ}$. Ultimately, $0^{\circ}$ is considered the best choice for developing the final regimen.

\section{Result of clinical trial}

\subsection{Baseline characteristics}

There were no significant differences in baseline date between two groups, such as age, weight and the time of the injury (all $P>0.05$, date are shown in Table 2 ).

\subsection{Global Function}

\subsubsection{DASH Scores}

The DASH questionnaire is used to grade disability from the nerve injury. The DASH scores did not exhibit any significant differences among the groups before the treatment. After 5 weeks, the subjects have an average $67.29 \pm 11.90$ score in experimental group and decline to $47.99 \pm 13.54$ score $(P<0.01)$. The mean DASH scores before treatment in the control group was $72.12 \pm 14.77$, and the mean scores after treatment was $66.36 \pm 20.43$ ( $P>0.05)$. The experimental group participants had a mean scores loss of $29 \%$ at 5 weeks; the control group lost $8 \%$. Differences of comparison within groups were statistically

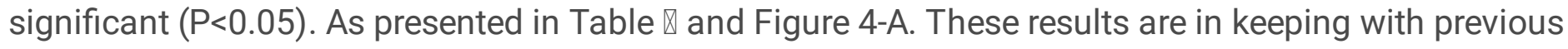
observational studies, which Tang think direct electrical stimulation by acupuncture needles improved patient's DASH scores and could returned to work around the 3 month of the treatment (Tang et al., 2016). Hence, we believe percutaneous nerve stimulation regimen can reduce the degree of disability and improve patients forearm functional activity. We guess this regimen may help restoring median neural functions, thereby actually improving overall functionality of the upper limb.

\subsubsection{Functional Score of Median Nerve}


According to the evaluation standard of the upper limb part of the Chinese Medical Association, the median neurological function of the patients was evaluated before and after treatment. After 5 weeks treatment, the scores of the functional score of median nerve increased by $24 \%$ in the experimental group and by $22 \%$ in the control group and the differences are statistically significant (experimental group: $\mathrm{P}=0.005$; control group: $\mathrm{P}=0.002$ ). Analyzing the data of these analyses, it can be seen that before treatment the score is no significant in two groups $(P=0.074)$. However, compared with the control group, the score of the experimental group is 1.3 times higher than that of the control group after treatment. The between-groups contrast after treatment show significant result between the two groups $(P=0.046)$. As presented in Table 2 and Figure 4-B. The neurological function of median is significantly ameliorated by both interventions. In contrast, the percutaneous nerve stimulation regimen showed better effect compared with transcutaneous electric nerve stimulation. The results confirmed our previous speculation which restoration of neurological function contributed to the overall functionality of the upper limb. We think the improvement of upper limb function is largely correlated with the reinnervation. However, the assessments of overall function are relatively general and vague. Thus a specific measurement of the condition of median nerve regeneration would be required such as sensory and motor function.

\subsection{BMRC of Sensory}

The BMRC score divide sensory function into eight grades (S0-S4). Recovery above grade S2 is considered good. Therapists evaluate patients according to deep sensation, superficial sensory and twopoint discrimination. Subsequently, measures of treatment effect data will be analyzed. After treatment, there are 12 patients with grade $S 2$ or above in the experimental group and 7 patients in the control group after treatment. Within-group comparisons indicate that after 5 weeks of treatment, there is significant differences in experimental group $(P=0.009)$ and control group $(P=0.034)$. The between-groups contrast show the contrast revealed significant differences between groups after treatment for 5 weeks $(P=0.019)$. These results indicate that the experimental group has better sensory function after the intervention than the control group. Some previous studies argue that electrical stimulation can dramatically increase tactile discrimination and pressure detection (Wong et al., 2015), and lead to cutaneous regeneration of DRG neurons(Koetsier et al., 2020). Those previous clinical studies supported our results.

\subsection{Motor Function \\ 2.3.1 BMRC of Motor}

The BMRC score divide motor function into six grades (M0-M5). Recovery above grade M3 is considered good. Therapists evaluate patients according to their motor ability. After treatment, there were 10 patients with grade $\mathrm{M} 3$ or above in the experimental group and only 3 patients in the control group. Within-group comparisons indicate that after 5 weeks of treatment, there is significant differences both experimental group $(P=0.002)$ and control group $(P=0.025)$ in motor assessment. The between-groups contrast show the contrast revealed significant differences between groups after treatment for 5 weeks $(P=0.001)$. However, there is always some subjectivity involved in the process at the scale assessment. Thus, an objective method needs to be developed to evaluate motor function such as muscle strength. 


\subsubsection{Grip Strength}

Skeletal muscles, which control human movement, are innervated by the nervous system. Median nerve supplies a myotome of multiple hand muscles, so grip strength is used as the main objective index in our study. There were individual differences between patients, we use the grip strength divide into the weight to reduce individualization. Compared with before, the grip strength improved significantly in all the treatment groups. Intra-group comparisons indicate that after 5 weeks of treatment, there is significant differences both experimental group $(P=0.025)$ and control group $(P=0.014)$. Notably, percutaneous nerve stimulation regimen treatment resulted in an upregulation of grip strength by 2.6 -fold after 5 weeks. The between-groups contrast before treatment did not show any significant result between the two groups $(P=0.311)$; however, the grip strength improvement of experimental group is 4 times higher than that of control group, the contrast reveal significant differences between groups after treatment for 5 weeks $(P=0.042)$. As presented in Table 2 and Figure 4-C. These results further demonstrated that percutaneous nerve stimulation regimen can better promote nerve regeneration and reinnervation in atrophied muscles than TENS.

\section{Discussion}

$\mathrm{PNI}$ is a common disease in which damage to nerve endings or distal ends occurs, representing $2 \%$ of all extremity traumas, resulting in grievous and even long-term physical and functional disability (Robinson, 2000; Wang et al., 2019). And median nerve injury is the most common PNI in humans (Mansiz-Kaplan et al., 2020). Studies have revealed that wallerian degeneration is initiated immediately after PNI in the distal nerve stumps, suggesting that these axons denature and disintegrate. Thus, one aim of therapeutic treatments is to create a microenvironment that is conducive to axonal regrowth and reinnervation(Conforti et al., 2014). The effectiveness of the proposed regimen is verified by all the experimental results above. Outcome measures of the experimental group were better than those of the control group whether sensory and motor or global function. The reason for that might be that percutaneous nerve stimulation facilitates staggered axonal regeneration. Several studies mentioned electrical stimulation accelerate the regeneration of axon by increasing production of neurotrophins (such as BDNF, NT-3) and expression of regeneration related genes (English et al., 2007; Geremia et al., 2007). Some researchers have explored cellular mechanisms by which ES accelerates nerve regeneration. Raised cyclic adenosine monophosphate (CAMP) levels can increase the expression of regeneration-associated genes such as Ta1 tubulin and growth-associated protein-43. Cytoskeletal assembly is enhanced through activation of the cAMP response element binding (CREB) protein, regulation of Ta1 tubulin, and the inhibition of Rho, which is a protein in the $\mathrm{p} 75 \mathrm{NgR}$ receptor (p75-NgR) pathway(McGregor and English, 2018). CREB activation is induced by the mitogen-activated protein kinase (MAPK) pathway. When a specific p38 MAPK inhibitor is implemented, CREB activation and neurite outgrowth are also suppressed. Hence, it is believed that ES-induced activation of the p38 MAPK pathway may play an important role in promoting neurite outgrowth (Kawamura and Kano, 2019). But additionally, correct reinnervation is regarded as another reason for the therapeutic effects of ES. Stimulation not only accelerated the speed 
of neuroregeneration, but also reduced staggered regeneration in a mouse model of PNI(Al-Majed et al., $2000 \mathrm{~b}$ ). Further, the stimulation leads to a similar distribution of neurons in PNI mice and control mice. This occurs by redirecting neurons back to the tissue they served originally, rather than by redistributing them (Brushart et al., 2005). Franz et al.(Franz et al., 2008)also found that brief periods of ES could increase polysialic acid expression in regenerating axons. This could promote increases in collateral sprouting and in the size of arborization fields, which improve the exactness of axon growth.

Existing research mainly discusses form the viewpoint of physiology. This study also could to be viewed in the context of electrical. Our percutaneous nerve stimulation regimen what electrical needles are parallel to each other and located at both ends of the injury nerve with a distance of $3 \mathrm{~cm}$, creates an electric field parallel to the nerve and maximize the current density and active function. Ultimately, this may be one of the mechanisms that helps optimize treatment effect for patients undergoing median nerve injury. Furthermore, percutaneous nerve stimulation can transmit electricity subcutaneously through the skin and onward. This compensates for the disadvantage of TENS, and it has a low-cost advantage and required no implanted electrodes.

Furthermore, we will improve the clinical experiment plan in further research, such as expanding the sample size and observing the long-term effect. Additionally, this study was very selective in the sense that we selected patients only with median nerve rupture. Next, we still need to investigate whether the regimen will hold with other types of PNI.

\section{Conclusion}

In conclusion, we have presented a treatment regimen by element finite modeling for median nerve rupture. Our finite element simulation shows that percutaneous nerve stimulation regimen is parallel to each other; electrical needles are located at both ends of the injury nerve with a distance of $3 \mathrm{~cm}$. In order to verify the results of the above finite element model, the clinical experiments were carried out with the same parameters as the finite element simulation. Eventually, the effectiveness of the regimen is verified by experimental results. The patient had improvement in functional motor, sensory, and overall function after 5 weeks treatment. This suggests that the treatment regimen can effectively promote nerve regeneration and nerve re-innervation in patients with median nerve rupture after surgery, thus promoting the improvement of overall function. It is a potential clinical treatment regimen, which is worthy of clinical promotion and popularization.

\section{Abbreviations}

$\mathrm{PNI}$

peripheral nerve injury

ES

Electrical stimulation

BDNF 
brain derived neurotrophic factor

TENS

transcutaneous electrical nerve stimulation

SQS

subcutaneous electrical stimulation

PNS

peripheral nerve stimulation

EA

electroacupuncture

FEM

finite element model

BMRC

British Medical Research Council

DASH

disability of arm shoulder and hand

cAMP

cyclic adenosine monophosphate

CREB

cAMP response element binding

$\mathrm{p} 75-\mathrm{NgR}$

p75 NgR receptor

MAPK

mitogen-activated protein kinase.

\section{Declarations}

Acknowledgements: At the point of finishing this paper, l'd like to express my sincere thanks to all those who have lent me hands in the course of my writing this paper. I'd like to thank Shuai Wang, Yusong Han, Siyao Li, Jiaxin Li and Shiyi Zhang. Without their help, it would be much harder for me to finish this paper.

Conflict of interest: All authors claim that there are no conflicts of interest.

Author contributions: Author contributions: Author contributions: Xiaolei Chu and Xizi Song wrote the initial manuscript draft. Yuru Li, Ziren Wu, Qi Li and Qingwen Li contribute to the conception and design. Dong Ming and Xiaosong Gu critically revise the manuscript. All authors approve the final manuscript draft.

Availability of data and materials: Adequately deidentifed data used and/or analyzed during the current study are available from the corresponding author on reasonable request.

Ethics approval and consent to participate: The clinical trial involved in this study was approved by the Human Research Ethics Committee of Tianjin Hospital. All patients voluntarily signed written informed 
consent. This randomized controlled trial has been registered in the Chinese Clinical Trial Registry (ChiCTR2000030790).

Consent for publication: Not applicable.

Financial support: This work was supported by grants from the National Natural Science Foundation of China (No. 81801787), China Postdoctoral Science Foundation (2018M640238), Natural Science Foundation of Tianjin City (20JCQNJC01690).

\section{References}

Al-Majed AA, Brushart TM, Gordon T (2000a) Electrical stimulation accelerates and increases expression of BDNF and trkB mRNA in regenerating rat femoral motoneurons. Eur $\mathrm{J}$ Neurosci 12:4381-4390.

Al-Majed AA, Neumann CM, Brushart TM, Gordon T (2000b) Brief electrical stimulation promotes the speed and accuracy of motor axonal regeneration. J Neurosci 20:2602-2608.

Brushart TM, Jari R, Verge V, Rohde C, Gordon T (2005) Electrical stimulation restores the specificity of sensory axon regeneration. Exp Neurol 194:221-229.

Brushart TM, Hoffman PN, Royall RM, Murinson BB, Witzel C, Gordon T (2002) Electrical stimulation promotes motoneuron regeneration without increasing its speed or conditioning the neuron. $\mathrm{J}$ Neurosci 22:6631-6638.

Bunn SJ, Lai A, Li J (2019) DC Electric Fields Induce Perpendicular Alignment and Enhanced Migration in Schwann Cell Cultures. Ann Biomed Eng 47:1584-1595.

Cavalcante Miranda de Assis D, Martins Lima Ê, Teixeira Goes B, Zugaib Cavalcanti J, Barbosa Paixão A, Vannier-Santos MA, Martinez AM, Baptista AF (2014) The parameters of transcutaneous electrical nerve stimulation are critical to its regenerative effects when applied just after a sciatic crush lesion in mice. Biomed Res Int 2014:572949.

Conforti L, Gilley J, Coleman MP (2014) Wallerian degeneration: an emerging axon death pathway linking injury and disease. Nat Rev Neurosci 15:394-409.

English AW, Schwartz G, Meador W, Sabatier MJ, Mulligan A (2007) Electrical stimulation promotes peripheral axon regeneration by enhanced neuronal neurotrophin signaling. Dev Neurobiol 67:158-172.

Franz CK, Rutishauser U, Rafuse VF (2008) Intrinsic neuronal properties control selective targeting of regenerating motoneurons. Brain 131:1492-1505.

Gabriel S, Lau RW, Gabriel C (1996) The dielectric properties of biological tissues: III. Parametric models for the dielectric spectrum of tissues. Phys Med Biol 41:2271-2293. 
Geremia NM, Gordon T, Brushart TM, Al-Majed AA, Verge VM (2007) Electrical stimulation promotes sensory neuron regeneration and growth-associated gene expression. Exp Neurol 205:347-359.

Gordon T, Udina E, Verge VM, de Chaves El (2009) Brief electrical stimulation accelerates axon regeneration in the peripheral nervous system and promotes sensory axon regeneration in the central nervous system. Motor Control 13:412-441.

Gordon T, Amirjani N, Edwards DC, Chan KM (2010) Brief post-surgical electrical stimulation accelerates axon regeneration and muscle reinnervation without affecting the functional measures in carpal tunnel syndrome patients. Exp Neurol 223:192-202.

Hassan Y, Eldosoky M, El-Wakad MTI (2018a) The effect of vascular diseases on bioimpedance measurements: mathematical modeling. Biomedical Research 5.

Hassan Y, Eldosoky MAA, El-Wakad MTI (2018b) The effect of vascular disease on bioimpedance measurements:mathematical modeling. Biomedical Research 5.

Hudak PL, Amadio PC, Bombardier C (1996) Development of an upper extremity outcome measure: the DASH (disabilities of the arm, shoulder and hand) [corrected]. The Upper Extremity Collaborative Group (UECG). Am J Ind Med 29:602-608.

Ju C, Park E, Kim T, Kim T, Kang M, Lee KS, Park SM (2020) Effectiveness of electrical stimulation on nerve regeneration after crush injury: Comparison between invasive and non-invasive stimulation. PLoS One 15:e0233531.

Kawamura K, Kano Y (2019) Electrical stimulation induces neurite outgrowth in PC12m3 cells via the p38 mitogen-activated protein kinase pathway. Neurosci Lett 698:81-84.

Kim J, Han SJ, Shin DH, Lee WS, Choi JY (2011) Subthreshold continuous electrical stimulation facilitates functional recovery of facial nerve after crush injury in rabbit. Muscle Nerve 43:251-258.

Koetsier E, Franken G, Debets J, Heijmans L, van Kuijk SMJ, Linderoth B, Joosten EA, Maino P (2020) Mechanism of dorsal root ganglion stimulation for pain relief in painful diabetic polyneuropathy is not dependent on GABA release in the dorsal horn of the spinal cord. CNS Neurosci Ther 26:136-143.

Koo J et al. (2018) Wireless bioresorbable electronic system enables sustained nonpharmacological neuroregenerative therapy. Nat Med 24:1830-1836.

Lal D, Hetzler LT, Sharma N, Wurster RD, Marzo SJ, Jones KJ, Foecking EM (2008) Electrical stimulation facilitates rat facial nerve recovery from a crush injury. Otolaryngol Head Neck Surg 139:68-73.

Liu YP, Luo ZR, Wang C, Cai H, Zhao TT, Li H, Shao SJ, Guo HD (2020) Electroacupuncture Promoted Nerve Repair After Peripheral Nerve Injury by Regulating miR-1b and Its Target Brain-Derived Neurotrophic Factor. Front Neurosci 14:525144. 
Lundborg G, Rosén B, Dahlin L, Holmberg J, Rosén I (2004) Tubular repair of the median or ulnar nerve in the human forearm: a 5-year follow-up. J Hand Surg Br 29:100-107.

Mansiz-Kaplan B, Pervane-Vural S, Gursoy K, Nacir B (2020) Median nerve conduction studies in rabbits. BMC Neurosci 21:34.

McGregor CE, English AW (2018) The Role of BDNF in Peripheral Nerve Regeneration: Activity-Dependent Treatments and Val66Met. Front Cell Neurosci 12:522.

McNeal DR (1976) Analysis of a model for excitation of myelinated nerve. IEEE Trans Biomed Eng 23:329-337.

Mukudai S, Matsuda KI, Nishio T, Sugiyama Y, Bando H, Hirota R, Sakaguchi H, Hisa Y, Kawata M (2015) Differential responses to steroid hormones in fibroblasts from the vocal fold, trachea, and esophagus. Endocrinology 156:1000-1009.

Novak CB, Anastakis DJ, Beaton DE, Katz J (2009) Patient-reported outcome after peripheral nerve injury. J Hand Surg Am 34:281-287.

Pan D, Gu Y, Shi D, Shou K (2000) Chinese Society of Hand Surgery upper limb functions assessment. Chin J Hand Surg 16:130-135.

Pang Y, Tang Y, Li Q, Xie J, Zang X, Liu T, Zhang B, Chen H (2013) [Effects of human umbilical cord mesenchymal stem cells on the proliferation of hematopoietic malignancies]. Xi Bao Yu Fen Zi Mian Yi Xue Za Zhi 29:714-718.

Park S, Liu CY, Ward PJ, Jaiswal PB, English AW (2019) Effects of Repeated 20-Hz Electrical Stimulation on Functional Recovery Following Peripheral Nerve Injury. Neurorehabil Neural Repair 33:775-784.

Power HA, Morhart MJ, Olson JL, Chan KM (2020) Postsurgical Electrical Stimulation Enhances Recovery Following Surgery for Severe Cubital Tunnel Syndrome: A Double-Blind Randomized Controlled Trial. Neurosurgery 86:769-777.

Rattay F, Aberham M (1993) Modeling axon membranes for functional electrical stimulation. IEEE Trans Biomed Eng 40:1201-1209.

Robinson LR (2000) Traumatic injury to peripheral nerves. Muscle Nerve 23:863-873.

Schreuders TA, Roebroeck M, van der Kar TJ, Soeters JN, Hovius SE, Stam HJ (2000) Strength of the intrinsic muscles of the hand measured with a hand-held dynamometer: reliability in patients with ulnar and median nerve paralysis. J Hand Surg Br 25:560-565.

Shen N, Zhu J (1996) Functional assessment of peripheral nerve injury and repair. J Reconstr Microsurg 12:153-157. 
Soldado F, Bertelli JA, Ghizoni MF (2016) High Median Nerve Injury: Motor and Sensory Nerve Transfers to Restore Function. Hand Clin 32:209-217.

Suslu H, Altun M, Erdivanli B, Turan Suslu H (2013) Comparison of the effects of local and systemic dexamethasone on the rat traumatic sciatic nerve model. Turk Neurosurg 23:623-629.

Tang YJ, Wu MH, Tai CJ (2016) Direct electrical stimulation on the injured ulnar nerve using acupuncture needles combined with rehabilitation accelerates nerve regeneration and functional recovery-A case report. Complement Ther Med 24:103-107.

Wachter NJ, Mentzel M, Krischak GD, Gülke J (2018a) Quantification of hand function by power grip and pinch strength force measurements in ulnar nerve lesion simulated by ulnar nerve block. J Hand Ther 31:524-529.

Wachter NJ, Mentzel M, Hütz R, Krischak GD, Gülke J (2018b) Quantification of weakness caused by distal median nerve lesion by dynamometry. $J$ Hand Ther 31:74-79.

Wan L, Xia R, Ding W (2010) Short-term low-frequency electrical stimulation enhanced remyelination of injured peripheral nerves by inducing the promyelination effect of brain-derived neurotrophic factor on Schwann cell polarization. J Neurosci Res 88:2578-2587.

Wang ML, Rivlin M, Graham JG, Beredjiklian PK (2019) Peripheral nerve injury, scarring, and recovery. Connect Tissue Res 60:3-9.

Wong JN, Olson JL, Morhart MJ, Chan KM (2015) Electrical stimulation enhances sensory recovery: a randomized controlled trial. Ann Neurol 77:996-1006.

Yu AP, Shen YJ, Qiu YQ, Li J, Shen YD, Wang XM, Cong M, He QR, Chen QZ, Jiang S (2019) Comparative effects of implanted electrodes with differing contact patterns on peripheral nerve regeneration and functional recovery. Neurosci Res 145:22-29.

Zhang M, Zhang Y, Bian Y, Fu H, Xu Y, Guo Y (2018) Effect of long-term electroacupuncture stimulation on recovery of sensorimotor function after peripheral nerve anastomosis. Acupunct Med 36:170-175.

Zhang X, Xin N, Tong L, Tong XJ (2013) Electrical stimulation enhances peripheral nerve regeneration after crush injury in rats. Mol Med Rep 7:1523-1527.

\section{Figures}


A

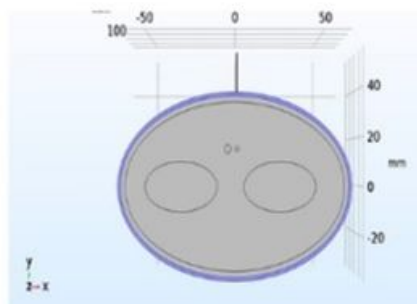

Skin

C

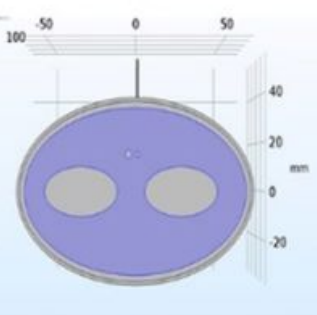

Skeletal Muscle

E

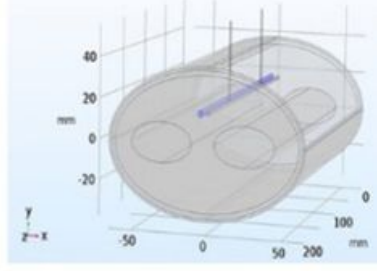

Blood Vessel
B

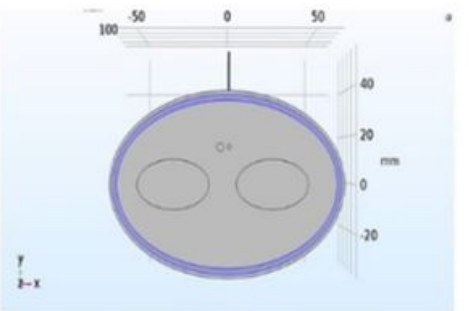

Fat

D

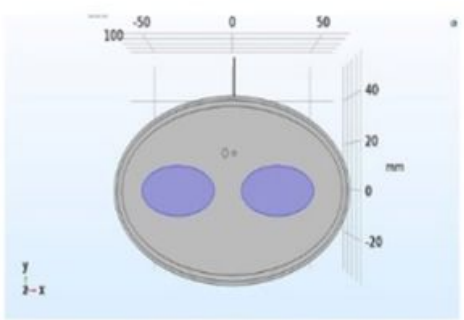

Bone

F

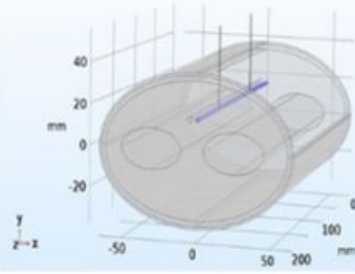

Nerve

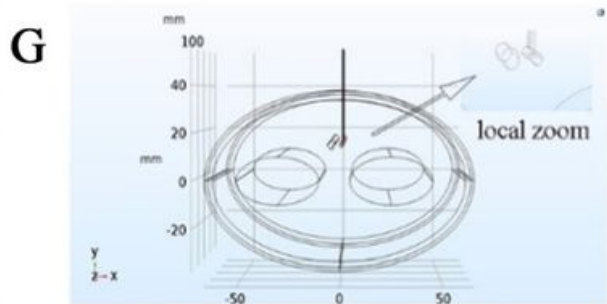

Central line of Nerve

$\mathrm{H}$

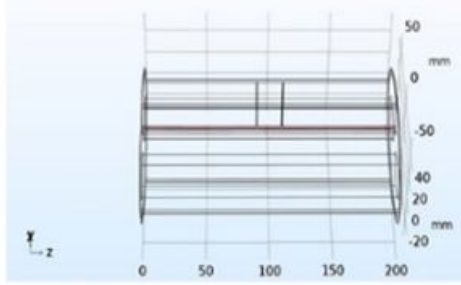

Forearm line of Nerve

I

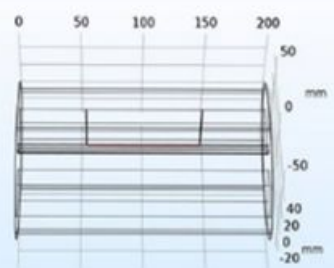

Line Between Two Needle

\section{Figure 1}

Geometry of the Human Forearm Model A: The blue parts of the model represent the skin; B: The blue parts of the model represent the fat; $\mathrm{C}$ : The blue parts of the model represent the skeletal muscle; $\mathrm{D}$ : The blue parts of the model represent the bone; $\mathrm{E}$ : The blue parts of the model represent the blood vessel; $\mathrm{F}$ : The blue parts of the model represent the nerve; G: The red line in the model is represented as the central line of nerve; $\mathrm{H}$ : The red line segment in the model is represented as the forearm line of nerve; $\mathrm{I}$ : The red line segment in the model is represented as the line between two needle. 


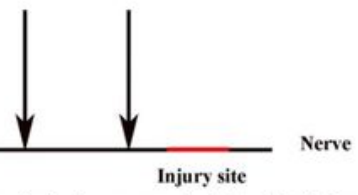

The electrical needle is place on proximal site of the injury nerve

Figure-B

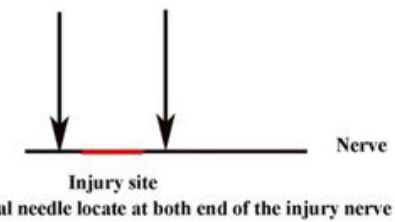

Figure-C
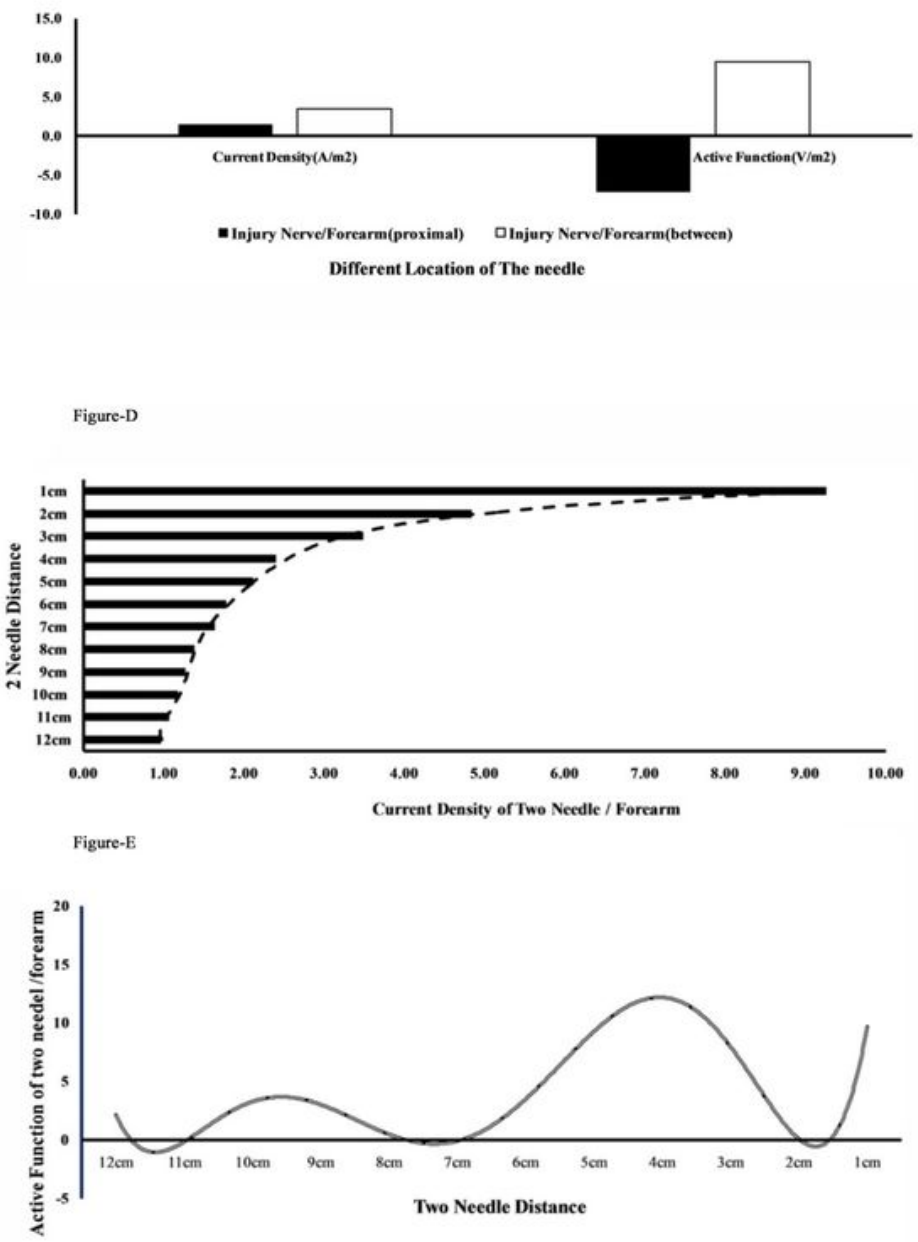

\section{Figure 2}

Locations and distance of the Needles A: The electrical needle is place on proximal site of the injury nerve: the black arrows represent needle electrode, the black segment represent nerve and the red segment represent injury site; $\mathrm{B}$ : The electrical needle location at both end of the injury nerve; $\mathrm{C}$ : Current density and action function at different location of the needle; D: Current density at different needle distance; E: Active function at different needle distance. 
Figure-A Current Density of Two Needle/Forearm

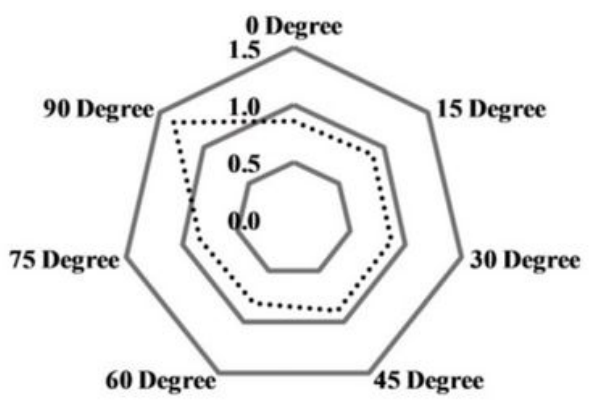

Figure-B Active Function of Two Needle/Forearm

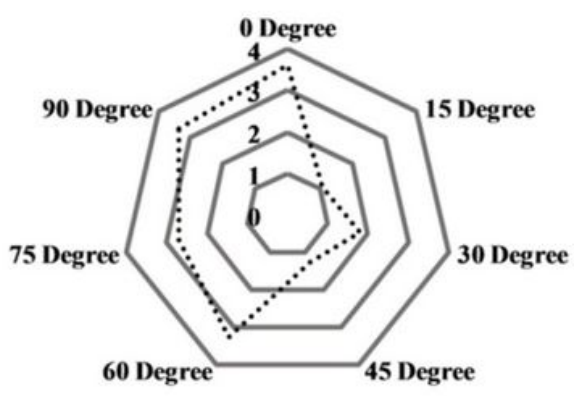

\section{Figure 3}

The Angle Between Two Needles A: Current density at Different Degree; B: Active function at Different Degree 
Figure-A

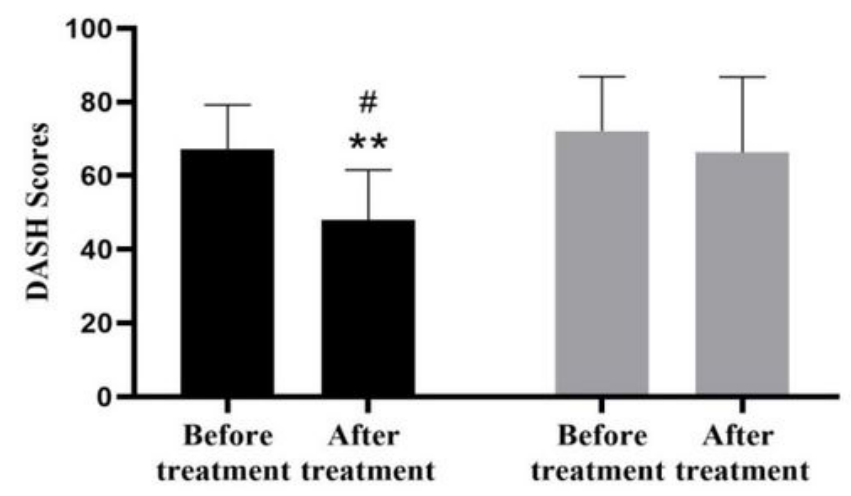

Figure-B

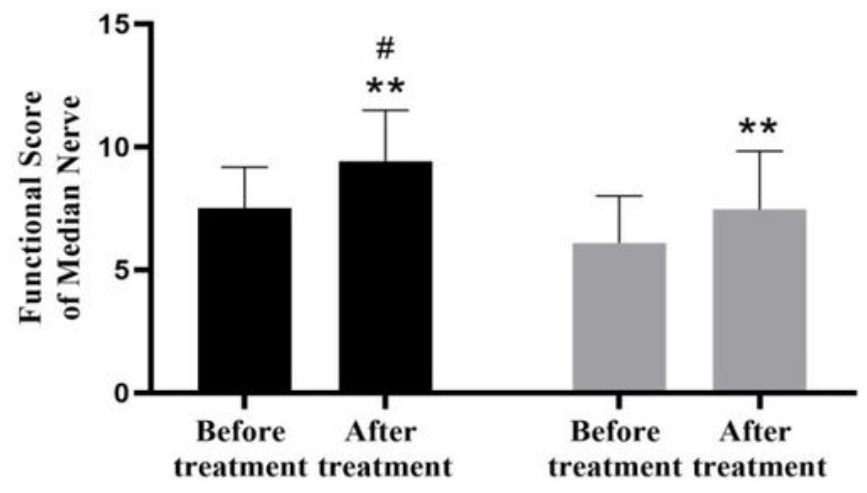

Figure-C

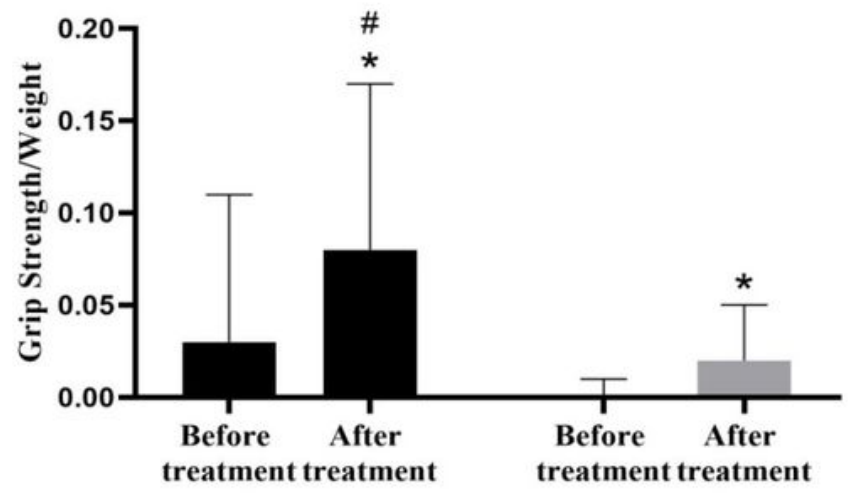

- Experimental group

- Control group
- Experimental group

Control group

\section{Figure 4}

Complete Histogram 1) A: Grip strength; B: Functional score of median nerve; C: DASH scores 2) * Intragroup comparison, $\mathrm{P}<0.05$;** Intra-group comparison, $\mathrm{P}<0.01$; \# Between-group comparisons, $\mathrm{P}<0.05$; \#\# Between-group comparisons, $\mathrm{P}<0.01$.

\section{Supplementary Files}


This is a list of supplementary files associated with this preprint. Click to download.

- Supplementary.docx 